\title{
Modeling of precipitation kinetics of TCP-phases in single crystal nickel-base superalloys
}

\author{
Ralf Rettig ${ }^{a}$, Astrid Heckl ${ }^{b}$ and Robert F. Singer ${ }^{c}$ \\ Institute of Science and Technology of Metals, Department of Materials Science and Engineering, \\ University of Erlangen, Martensstr. 5, D-91058 Erlangen, Germany \\ aralf.rettig@ww.uni-erlangen.de, bastrid.heckl@ww.uni-erlangen.de, \\ robert.singer@ww.uni-erlangen.de
}

\begin{abstract}
Key words: superalloys, CALPHAD, ThermoCalc, DICTRA, rhenium, ruthenium, precipitation model

Abstract. The precipitation of brittle so-called TCP-phases is critical for the application of Recontaining single crystal superalloys. In this work a fully multicomponent precipitation model is presented, which is capable of simulating the precipitation process of the TCP-phases in superalloys considering complex precipitation sequences with several metastable phases. The model is coupled to multicomponent thermodynamic CALPHAD calculations and relies on multicomponent diffusion models based on the TC-API interface of the software DICTRA. The required mobility database has been newly developed and covers all relevant alloying elements of the Ni-base superalloys including rhenium $(\mathrm{Re})$ and ruthenium $(\mathrm{Ru})$. It is well known that adding $\mathrm{Ru}$ strongly reduces TCP-phase precipitation. Based on the developed precipitation model, possible mechanisms are investigated to explain this effect and it is concluded that Ru mostly influences the nucleation rate by a combined influence on interface energy, "reverse partitioning" and $\gamma$ '-phase fraction.
\end{abstract}

\section{Introduction}

Nickel-base superalloys are widely applied for high temperature applications, especially for turbine blades in the hottest sections of industrial and aero-engine gas turbines [1-2]. Reduction of fuel consumption and engine emissions are very important design goals today. The efficiency of the gas turbine is determined by the burning temperature. Obviously, the material capability of the turbine blades limits this temperature. Therefore improvements in creep strength through material development as well as single-crystalline casting and application of thermal barrier coatings increase the turbine efficiency [3]. Nickel-base superalloys contain typically more than eight alloying elements. Although they have been under investigation for decades due to the complexity there is still space for improvements $[2,4]$.

In the last decade, major improvements in creep strength could be achieved by the development of the $3^{\text {rd }}$ and $4^{\text {th }}$ generation single crystalline superalloys, which contain up to $6 \mathrm{wt}-\%$ Re and $6 \mathrm{wt}-\%$ $\mathrm{Ru}$ [1]. Rhenium is known to be a very good solid solution hardener [5], nevertheless it strongly increases the susceptibility to brittle topologically-close packed (TCP) phase precipitation, which limits the acceptable content of rhenium [6-7]. One answer to this problem was to add ruthenium in the $4^{\text {th }}$ generation superalloys. This element very effectively reduces the TCP-phase precipitation and increases the creep strength [8-9]. The mechanism of this effect is still not well understood $[5,7,8,10]$. Both elements, $\mathrm{Re}$ as well as $\mathrm{Ru}$ are extremely expensive and there are doubts on the availability [11]. In order to optimize the alloy composition, the mechanism of the Ru-effect is investigated in this work with numerical methods. Modeling precipitation in superalloys is challenging because of the high number of alloying elements. Most of the multicomponent models developed in the past were applied to high temperature steels. The first, still simple models benefiting from the CALPHAD-method were by [12-13]. Later works by [14] were focused on TCP-phases in austenitic steels, but are hardly applicable to superalloys with more alloying elements because of the complexity of the algorithm. The phase-field method is still too challenging for long-term simulations of nickel-based superalloys. In the following, a model based on ideas from [15] will be applied. The advantage of this so-called numerical Kampmann-Wagner method is that it can be straightforwardly extended to multicomponent systems and that it is applicable to a large number of precipitates and therefore allows the calculation of complex precipitation sequences [14]. 


\section{Methods}

CALPHAD and diffusion modeling. The CALPHAD-method (Calculation of Phase Diagrams) allows the reliable calculation of thermodynamic properties of complex multicomponent systems such as compositions of phases, phase fractions and driving forces. It is based on the principle of Gibbs energy minimization. The CALPHAD approach has been extended to modelling multicomponent diffusion. In this work the TC-API programming library version 4 based on the software ThermoCalc version R and DICTRA version 24 (ThermoCalc, Stockholm, Sweden) is used. The thermodynamic database TTNi7 (ThermoTech Ltd, Surrey, UK) [16] as well as an inhouse diffusion database containing the element $\mathrm{Ru}$ were used. Recently, a verification of the database TTNi7 for Re- and Ru-containing superalloys was published by the present authors [17] and previous work already examined the quality for Re-containing alloys [18-19].

Multicomponent precipitation model. The developed precipitation model is based on the numerical Kampmann-Wagner method [15], which was originally developed for binary systems. It allows the simple calculation of precipitation sequences and is in this work extended to multicomponent alloys. A simple flowchart of the algorithm is presented in Fig. 1.

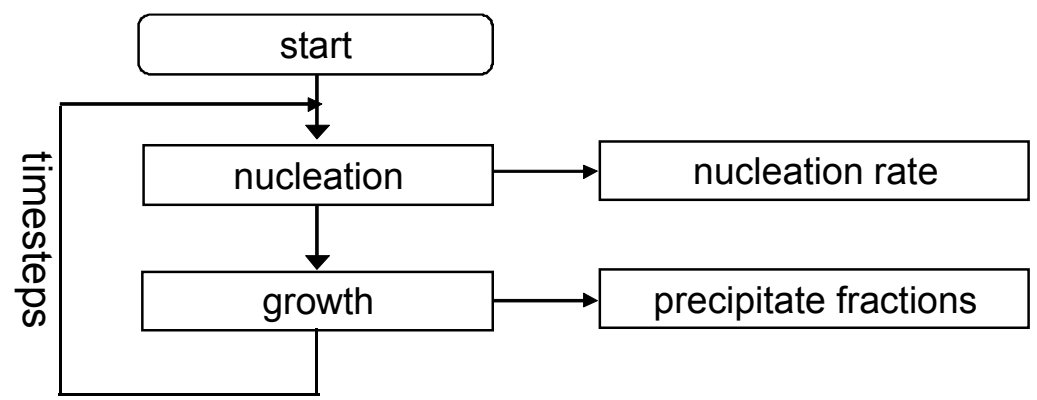

Figure 1: Schematic multicomponent numerical Kampmann-Wagner model implemented with MATLAB in this work.

The basic principle of this model is to separate all precipitates into a limited number of precipitate classes. In each class, a defined number of precipitates is stored. All precipitates in one class have identical size and chemical composition, because they are nucleated at the same time. In each timestep, at first the current nucleation rate is evaluated and if required, a new precipitate class is created. In a second step, the growth of all already existing precipitate classes is calculated with a multicomponent growth law. Details about this can be found in a forthcoming publication.

Nucleation model. The classical nucleation model was applied for calculating nucleation. Although the model has to cope with multicomponent nucleation, there still is no generally applicable multicomponent nucleation theory available yet. Despite that fact, most models in the literature were developed successfully by applying the classical nucleation law for multicomponent systems. In most cases the Gibbs free enthalpy $\Delta G_{v}$ of the precipitate is calculated using the CALPHADmethod $[14,20]$. The classical nucleation law is based on the overall Gibbs free energy $\Delta G$ of the precipitate, which consists of the chemical driving force $\Delta G_{v}$, the interface energy $\gamma_{i}$ of all interfaces $i$ and the misfit stress enthalpy $\Delta G_{S}$ :

$$
\Delta G=-V \cdot \Delta G_{v}+\sum_{i} \gamma_{i} A_{i}+V \cdot \Delta G_{s} \cdot f_{\text {het }}
$$

$V$ is the precipitate volume and $f_{h e t}$ is a factor considering the reduced misfit stress for heterogeneous nucleation. Application of this equation gives the following relation for the free enthalpy $\Delta G^{*}$ of a nucleate with the critical size:

$$
\Delta G^{*}=\frac{16 \pi \gamma^{3}}{3 \cdot\left(\Delta G_{v}-\Delta G_{s} \cdot f_{\text {het }}\right)^{2}}
$$

The overall nucleation rate $d N / d t$ of the precipitate at time $t$ can finally be obtained with $N(t)$ being the number of nucleates, $\mathrm{k}$ the Boltzmann constant, $N_{0}$ the density of nucleation sites, $\omega$ the frequency factor and $\Delta G_{t}$ the activation energy for Brownian motion [20]: 


$$
\frac{d N}{d t}(t)=\left(1-\frac{N(t)}{N_{0}}\right) \cdot\left(\omega \cdot N_{0} \cdot \exp \left(-\frac{\Delta G_{t}}{\mathrm{k} T}\right) \cdot \exp \left(-\frac{\Delta G^{*}\left(c_{i}(t)\right)}{\mathrm{k} T}\right)\right)
$$

This equation is applicable to multicomponent systems if the concentration-dependent activation energy $\Delta G^{*}\left(c_{i}\right)$ is calculated using the CALPHAD-method.

Growth model. A multicomponent growth model is presented based on the work from [21]. The state of the interface is determined on the one hand by the thermodynamic equilibrium described with the chemical potentials of precipitate $\mu_{P}^{i}$ and the matrix at the interface $\mu_{I}^{i}$ :

$$
\mu_{P}^{i}=\mu_{I}^{i}
$$

On the other hand, flux balance is valid for all elements $i$. The flux balance equation can be expressed for each element by:

$$
F(\phi)^{-1} \cdot v \cdot\left(c_{P}^{i}-c_{I}^{i}\right)=c_{I}^{i} M_{i} \frac{\mu_{M}^{i}-\mu_{I}^{i}}{\xi_{i} r}
$$

The growth rate is given by $v$, the diffusion mobility by $M_{i}$, the precipitate radius by $r$ and the chemical potential of the matrix phase by $\mu_{M}^{i}$. In Eq. $5 \xi_{i}$ is the proportionality constant connecting the precipitate radius to the size of the depleted zone in the matrix, i.e. $d_{i}=\xi_{i} r$. The non-linear equation system of Eq. 4-5 has to be solved for each timestep to obtain the growth rate and interface concentrations. Details can be found in [21] and a forthcoming publication. TCP-phases typically show a plate-like morphology, appearing needle-like in cross-sections. The precipitation model up to now only considers a spherical precipitate morphology. Based on the work by [22], a shape factor $F(\phi)$ is introduced in Eq. 5, which converts the spherical growth rate to that of a cylindrical precipitate with the aspect ratio $\phi=l /(2 \cdot r)$ and precipitate length $l$ and radius $r$ :

$$
F(\phi)=0.976 \cdot \phi^{-0.664}
$$

The matrix is depleted or enriched by the precipitation. Thus, the driving force for precipitation is reduced until equilibrium is reached. Our model performs a steady update of the matrix concentration $c_{M}^{i}$. The initial matrix concentration is $c_{0}^{i}$ and the precipitate fraction of $j$ is $V_{P}^{j}$.

$$
c_{M}^{i}(t)=\frac{c_{0}^{i}-\sum_{j}\left[\int_{0}^{V_{P}^{j}} c_{P, j}^{i}(V(t)) \cdot d V\right]}{1-\sum_{j} V_{P}^{j}(t)}
$$

All chosen parameters for the precipitation model in this work are summarized in Table 1.

Table 1: Full list of model parameters used for the precipitation simulations in this work.

\begin{tabular}{lllllllll}
\hline Fig. & $\boldsymbol{N}_{\boldsymbol{0}}\left[\mathbf{m}^{-3}\right]$ & $\boldsymbol{\gamma}\left[\mathbf{J m}^{-2}\right]$ & $\boldsymbol{G}_{\boldsymbol{t}}\left[\mathbf{k J ~ \mathbf { ~ m o l } ^ { - 1 } ]}\right.$ & $\boldsymbol{\Phi}[-]$ & $\boldsymbol{\varepsilon}[\mathbf{\%}]$ & $\boldsymbol{G}[\mathbf{G P a}]$ & $\boldsymbol{f}_{\text {het }}[-]$ & alloy \\
\hline 3 & $1 \cdot 10^{13}$ & 0.05 & 255 & 1 & 3.6 & 70 & 0.2 & Ni-12Cr-16W (at-\%) \\
4 & $1 \cdot 10^{15}$ & 0.05 & 255 & 200 & 3.6 & 70 & 0.2 & ASTRA1-10
\end{tabular}

\section{Results}

Reverse partitioning effect. "Reverse partitioning" describes the observation that adding ruthenium pushes more rhenium into the $\gamma$ '-phase. This reduces the Re-concentration in the matrix phase. In other words, the partitioning coefficient $k_{\mathrm{Re}}^{\gamma / \gamma^{\prime}}$ shall be reduced by adding $\mathrm{Ru}$. Re is the most important TCP-phase forming element. It has been controversially debated for a long time, whether this effect is responsible for the TCP-phase fraction reduction in $4^{\text {th }}$ generation superalloys. Nevertheless beside many works claiming the existence of this effect (for example [8,23]), it could not be found in many other alloys (for example [10]). In the following a thermodynamic study with ThermoCalc is presented, which investigates the combined influence of $\mathrm{Ru}$ and other alloying element on the partitioning coefficient of Re (see Fig. 2). 


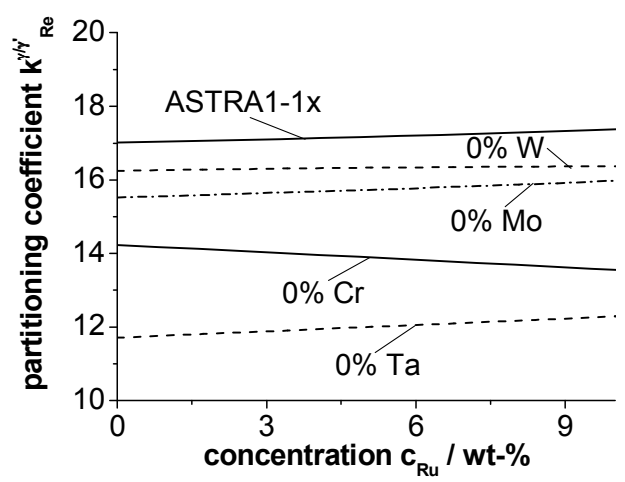

Figure 2: Calculated $\gamma / \gamma^{\prime}$-partitioning coefficient of Re in alloys based on the superalloys ASTRA1-1x (6.0Al-8.7Co5.1Cr-1.0Mo-3.1Re-xRu-6.6Ta-6.1W-Ni, wt-\%) in dependence of the Ru- and the Cr-, Mo-, Ta- and W-content. All elements have a large influence on the $\gamma / \gamma^{\prime}$-partitioning coefficient of Re. The element $\mathrm{Cr}$ determines, if "reverse partitioning" is present. This is recognized from negative slopes of the curves. The comparison to experimental results shows, that the calculated values are about a factor of 3 higher than the measured, but the relative changes are valid anyway.

The slope of the curves in Fig. 2 shows whether there is a "reverse partitioning" effect. A negative slope (for example for $0 \mathrm{wt}-\% \mathrm{Cr}$ ) in Fig. 2 is equivalent to "reverse partitioning". In fact, Fig. 2 clearly shows that not only Ru changes the partitioning behavior of Re, but that many other alloying elements determine the absolute value and if there is "reverse partitioning" or not. "Reverse partitioning" is obviously induced only for small $\mathrm{Cr}$-concentrations. Overall, W, Mo, Cr and Ta all significantly change the partitioning coefficient of Re. The values in Fig. 2 should not be taken as fully quantitative, because the present authors showed in [17] that the $\gamma$-concentration of $\operatorname{Re}$ calculated with TTNi7 typically has a systematic shift. Nevertheless, we are convinced that the relative changes are modeled correctly.

Precipitation model. At first the developed precipitation model is applied to a simple Ni-Cr-Wmodel alloy in order to check the basic model physics in a ternary (i.e. already multicomponent) system.

a)

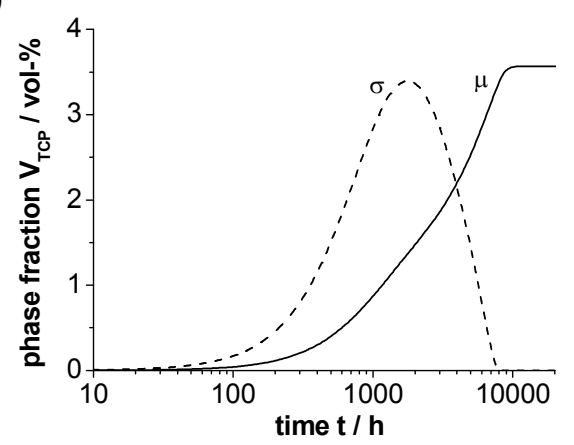

b)

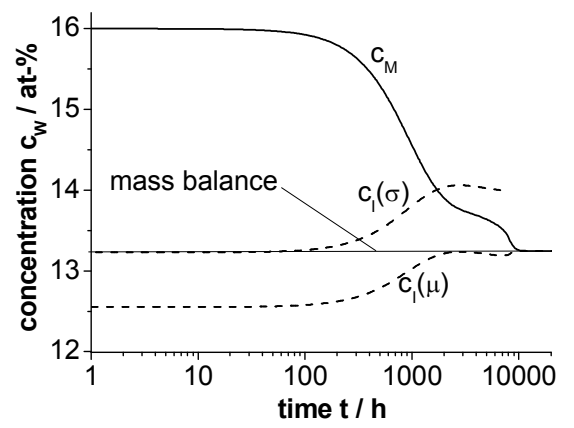

Figure 3: Precipitation sequence of the $\sigma$ - and the $\mu$-phase in the $\mathrm{Ni}-12 \mathrm{Cr}-16 \mathrm{~W}$ (at- $\%$ ) model alloy at $1050{ }^{\circ} \mathrm{C}$. The $\sigma$ phase is dissolved after some time. (a) Volume fractions of the precipitates, (b) concentrations of W in matrix $c_{M}$ and interface $c_{I}$ in dependence of time.

In the further sections as well as in a forthcoming publication, it will be shown that the model is fully capable of simulating real superalloys. Fig. 3a presents the precipitate phase fraction in the model alloy $\mathrm{Ni}-12 \mathrm{Cr}-16 \mathrm{~W}$ (at-\%) assuming only the $\sigma$ - and the $\mu$-phase being present. The $\sigma$-phase is precipitating as the first - obviously metastable - phase and it is dissolved later, when the stable $\mu$-phase starts to precipitate. After about 10000 hours, equilibrium with a volume fraction consistent to ThermoCalc calculations is reached. Fig. $3 \mathrm{~b}$ shows the related concentrations. As soon as a significant phase fraction of precipitates is formed, the matrix starts to get depleted.

According to Eq. 5, a phase will grow until the matrix concentration drops below the interface concentration - then it will be dissolved. Fig. $3 b$ shows this behavior for the $\sigma$-phase. When the system finally reaches equilibrium, the matrix concentration has reached a stable balance. 
Numerical study on the influence of ruthenium. In the following, different possible mechanisms for the Ru-effect on TCP-phases will be discussed based on the developed model. It is most often attributed to either a change in the $\gamma^{\prime}$-phase fraction $f_{\gamma^{\prime}}$ or the $\gamma / \gamma^{\prime}$-partitioning ratio $k_{R e}^{\gamma / \gamma^{\prime}}$ on addition of $\mathrm{Ru}$ [7]. The Re-matrix concentration $c_{\mathrm{Re}}^{\gamma}$, which is needed in the precipitation simulations (and all other concentrations), can be calculated using the Blavette-equation from the $\gamma^{\prime}$-phase fraction $f_{\gamma^{\prime}}$ and the $\gamma / \gamma^{\prime}$-partitioning ratio $k_{i}=c_{i}^{\gamma} / c_{i}^{\gamma^{\prime}}$ :

$$
c_{R e}^{\gamma}=k_{\mathrm{Re}} \cdot \frac{c_{R e}}{f_{\gamma^{\prime}}+k_{\mathrm{Re}} \cdot\left(1-f_{\gamma^{\prime}}\right)}
$$

The nominal Re-concentration is given by $c_{R e} . \gamma^{\prime}$-phase fraction and $\gamma / \gamma^{\prime}$-partitioning ratio can be determined from CALPHAD-calculations. In this section they will be changed artificially in order to explore their influence on TCP-phase precipitation. The CALPHAD- $\gamma^{\prime}$-phase fraction was reduced by $5 \mathrm{~mol}-\%$ and the $\gamma / \gamma^{\prime}$-partitioning ratio of Re to one third. The resulting hypothetical Re-content in the matrix was then calculated with Eq. 8 and included in the precipitation simulations.

a)

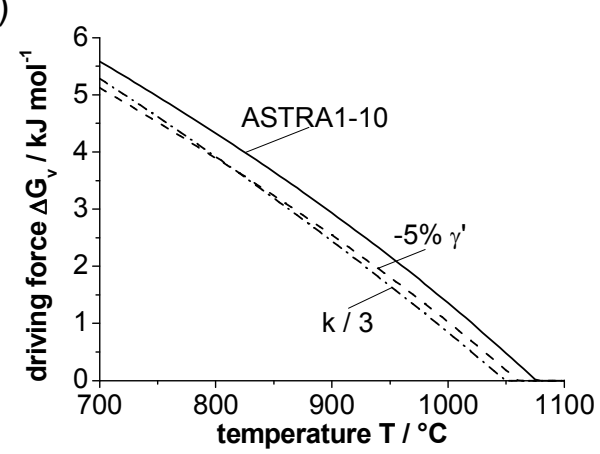

b)

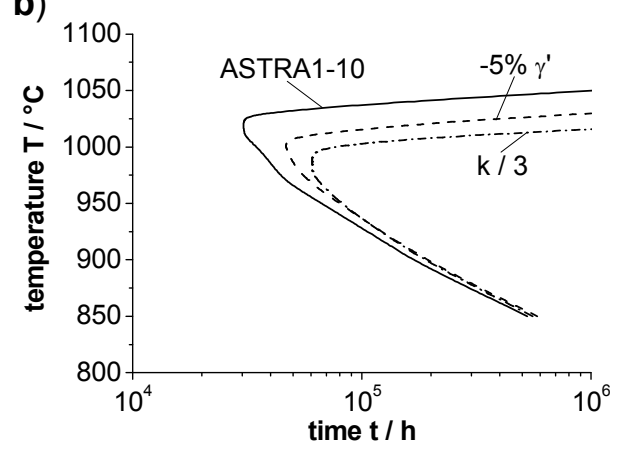

Figure 4: Numerical study on the separate influence of $\gamma / \gamma^{\prime}$-partitioning ratio and $\gamma^{\prime}$-phase fraction on the $\sigma$-phase in the alloy ASTRA1-10. (a) Driving force, (b) TTT-diagram for 0.5 vol- $\% \sigma$-phase precipitation. The composition of

ASTRA1-10 is given in the description of Fig. 2.

The results in Fig. 4a clearly show that the driving force for TCP-phase precipitation is reduced by both changes. Based on literature data, we assume that the chosen parameter changes represent the maximum possible effect of Ru. From Fig. $4 \mathrm{~b}$ it becomes clear that consequently the TTT-diagram for the TCP-precipitation is moved towards lower temperatures and longer times.

\section{Discussion}

The results presented in the previous section show that the "reverse partitioning" effect can occur in superalloys, but the situation is much more complex than considered before. A "reverse partitioning" effect could only be found in the calculations if the concentration of $\mathrm{Cr}$ was low. This is in agreement with [24] being the first work to experimentally show that a low Cr-content is a prerequisite for observing the "reverse partitioning" effect in the alloys they investigated. This result and the fact that Mo, Ta and $\mathrm{Ti}$ also change the $\gamma / \gamma^{\prime}$-partitioning ratio, explain the large variety of results in literature which were obtained for very different alloys. [7] discusses possible reasons for the impact of $\mathrm{Ru}$ on the nucleation rate of the TCP-phase precipitation. Ru might influence the $\gamma^{\prime}-$ phase fraction, the $\gamma / \gamma^{\prime}$-partitioning ("reverse partitioning") and the interface energy between matrix and precipitate. The theoretical study in this work indicates that the $\gamma$ '-phase fraction as well as the $\gamma / \gamma^{\prime}$-partitioning have an influence on the TCP-phase precipitation process - if they are influenced by $\mathrm{Ru}$. Nevertheless, the simulated effect is too small to be the only reason for the Ru-influence. The assumed changes of the two properties in the simulations are estimated to be the upper limit of a possible Ru-influence. Additionally, it is still under dispute whether Ru really influences the $\gamma^{\prime}-$ phase fraction or the $\gamma / \gamma^{\prime}$-partitioning ratio. For example, [7] found a reduction of the $\gamma^{\prime}$-phase fraction by $\mathrm{Ru}$, while $[5,25]$ could not find any in other alloys. It was shown in the previous section that the "reverse partitioning" effect is alloy dependent and this probably also holds for the $\gamma$ '-phase 
fraction. Further research, which could not be presented here, indicates that Ru has only a small influence on the diffusion controlled growth step. Due to that, we propose that there is a further influence of $\mathrm{Ru}$, which is postulated to be an increase in the interface energy between matrix and TCP-precipitate in Ru-containing alloys.

\section{Conclusions}

In this work a fully multicomponent precipitation model capable of simulating the complex precipitation process of TCP-phases in superalloys including precipitation sequences was developed. It was shown from thermodynamic studies that the so-called "reverse partitioning" depends on the concentration of other alloying elements, especially $\mathrm{Cr}$, Mo, Ta and $\mathrm{W}$. The $\gamma$ '-phase fraction and "reverse partitioning" have an influence on precipitation, but only the postulation of an influence of $\mathrm{Ru}$ on the matrix / precipitate interface energy can explain the huge effect of $\mathrm{Ru}$.

\section{Acknowledgements}

This work has received funds from the German Science Foundation (DFG) for the project MM3 in the framework of the Research Training Group 1229/1 "Stable and Metastable Multiphase Systems for High Temperature Applications".

\section{References}

[1] H. Harada, in: International Gas Turbine Congress, edited by M. Ito et al., Tokyo, Japan (2003), p. 1.

[2] C.M.F. Rae: Mater. Sci. Tech. 25 (2009) p. 479.

[3] B.B. Seth, in: Superalloys 2000, edited by T. M. Pollock et al., TMS, Seven Springs (2000), p. 3.

[4] R.C. Reed, T. Tao and N. Warnken: Acta Mater. 57 (2009) p. 5898.

[5] S. Neumeier, F. Pyczak and M. Göken, in: Superalloys 2008, edited by R. C. Reed et al., TMS, Seven Springs (2008), p. 109.

[6] C.M.F. Rae and R.C. Reed: Acta Mater. 49 (2001) p. 4113.

[7] R. Hobbs, L. Zhang, C.M.F. Rae and S. Tin: Met. and Mater. Trans. A 39 (2008) p. 1014.

[8] K. O'Hara, W. Walston, E. Ross and R. Darolia, United States Patent 5.482.789 (1996).

[9] A.C. Yeh, C.M.F. Rae and S. Tin, in: Superalloys 2004, edited by K. A. Green et al., TMS, Seven Springs (2004), p. 677.

[10]A. Volek, F. Pyczak, R.F. Singer and H. Mughrabi: Scripta Mater. 52 (2005) p. 141.

[11]P.J. Fink, J.L. Miller and D.G. Konitzer: JOM 62 (2010) p. 55.

[12] J.D. Robson and H.K.D.H. Bhadeshia: Mater. Sci. Tech. 13 (1997) p. 631.

[13] J.D. Robson and H.K.D.H. Bhadeshia: Mater. Sci. Tech. 13 (1997) p. 640.

[14] T. Sourmail and H.K.D.H. Bhadeshia: CALPHAD 27 (2003) p. 169.

[15] R. Kampmann, and R. Wagner, in: Acta-Scrip. Met., Conference Proceedings II, edited by P. Haasen et al., Pergamon, Oxford, (1984), p. 91.

[16] N. Saunders, M. Fahrmann and C. Small, in: Superalloys 2000, edited by K. Green et al., TMS, Seven Springs (2000), p. 803.

[17] R. Rettig, A. Heckl, S. Neumeier, F. Pyczak, M. Göken and R.F. Singer: Defect and Diffusion Forum 289-292 (2009) p. 101.

[18]E.H. Copland, N.S. Jacobson and F.J. Ritzert: Technical Memorandum NASA/TM-2001210897, NASA, Glenn Research Center (2001).

[19] J.C. Zhao and M.F. Henry: Adv. Eng. Mater. 4 (2002) p. 501.

[20]H. Sieurin and R. Sandström: Mater. Sci. Eng. A 444 (2007) p. 271.

[21] Q. Chen, J. Jeppson and J. Ågren: Acta Mater. 56 (2008) p. 1890.

[22] E. Kozeschnik, J. Svoboda and F.D. Fischer: Mater. Sci. Eng. A 441 (2006) p. 68.

[23] A.P. Ofori, C.J. Humphreys, S. Tin and C.N. Jones, in: Superalloys 2004, edited by K. A. Green et al., TMS, Seven Springs (2004), p. 787.

[24]L. Carroll, Q. Feng, J. Mansfield and T. Pollock: Met. Mater. Trans. 37A (2006) p. 2927.

[25]R.C. Reed, A.C. Yeh, S. Tin, S.S. Babu and M.K. Miller: Scripta Mater. 51 (2004) p. 327. 